\begin{abstract}
A FREQUENCY-DOMAIN TECHNIQUE FOR SEPARATING THE APERIODIC AND PERIODIC RESPONSE OF A IINEAR, LUMPED-PARAMETER SYSTEM EXCITED BY A PERIODIC DRIVING FUNCTION
\end{abstract}

\author{
B. R. Sandberg
}

November 20,1972

\begin{abstract}
Purpose
Occasionally one is interested in the steady-state values of the peak-to-peak ripple and the average response of a system excited by a periodic driving function. Also, the build-up of this response to the steady state is sometimes a point of interest. The author's problem was finding the maximum, peak-to-peak, steady-state ripple current in a quadrupole magnet driven by a six-pulse, SCR, bridge rectifier. The solution to this problem is treated in the examples (III) below.
\end{abstract}

\title{
Method
}

This type of problem can be solved rather easily by a technique in the complex frequency domain. Essentially the principal parts of the Laurent expansions of $\mathrm{Y}(\mathrm{s})$ about certain poles of this function are summed together forming the aperiodic response function $A(s)$. This $A(s)$ function has terms of the form $\frac{a_{k}}{\left(s+s_{0}\right)^{n}}$, which are valid everywhere in the complex plane except at $\mathbf{s}=-\mathbf{s}_{0}$. These terms transform to the time domain as 


$$
\frac{a_{K}}{(n-1) !} t^{n-1} e^{-s_{0} t}
$$

Having found $A(s)$, it is subtracted from $Y(s)$ leaving a function $P(s)$, which is the periodic response function.

It remains to describe which poles of $Y(s)$ are to be used to obtain the summation of the principal parts of the Laurent expansions. Let $\mathrm{H}(\mathrm{s})$, a rational function, be the impulse response function of the linear, lumped parameter, zero initial energy system--i.e. $H(s)=\frac{Y(s)}{X(s)}$ where $X(s)$ is the driving function. Therefore, $\mathrm{Y}(\mathrm{s})=\mathrm{H}(\mathrm{s}) \mathrm{X}(\mathrm{s})$ where:

$$
\begin{gathered}
x(s)=\frac{x_{0}(s)}{1-e^{-s T}} \\
\left.x_{0}(s)=x_{0}(s)\right]=\int_{0}^{T} x_{0}(t) e^{-s t} a t \\
x_{0}(t)=\left\{\begin{array}{cc}
x(t): & 0 \leq t \leq T \\
0: & t<0 \text { and } t>T
\end{array}\right. \\
s=\sigma+j \omega ; \quad T=\text { the period. }
\end{gathered}
$$

Thus we have:

$$
Y(s)=\frac{H(s) X_{0}(s)}{\left(1-e^{-s^{T}}\right)} \text {. }
$$

The poles of $\mathrm{Y}(\mathrm{s})$ about which Laurent expansions are to be made are those of $H(s)$, those of $x_{0}(s)$, and the simple pole of $\frac{1}{\left(1-e^{-s T}\right)}$ at $s=0 . H(s) X_{0}(s)$ may also have poles at $s=0$. The principal part of the Laurent expansion about the poles at $\mathbf{s}=0$ yield the 
aperiodic time domain terms that are non-zero as time approaches infinity (the steady-state average value), while all other principal parts yield the aperiodic time domain terms that go to zero as time approaches infinity.

A typical Laurent expansion about a pole of order $n$ at $s=-s_{0}$ looks like:

$$
f(s)=\underbrace{\frac{a_{-n}}{\left(s+s_{0}\right)^{-1}}+\frac{a_{-}(n-1)}{\left(s+s_{0}\right)^{n-1}}+\cdots+\frac{a_{-1}}{\left(s+s_{0}\right)^{1}}+a_{0}+\frac{a_{1}\left(s+s_{0}\right)^{1}}{1}+a_{2}\left(s+s_{0}\right)^{2}+\cdots}_{\begin{array}{l}
\text { The principal parts valid } \\
\text { at all s except } s=-s_{0}
\end{array}}
$$

For all poles of $H(s) X_{0}(s)$ not at zero, the following formulas are valid and simple to use for the coefficients of the principal parts of the Laurent expansion of $\mathrm{Y}(\mathrm{s})$ about them:

$$
\begin{aligned}
& a_{-1}=\left.\frac{1}{(n-1) !} \frac{d^{n-1}}{d s^{n-1}}\left[\left(s+s_{0}\right)^{n} f(s)\right]\right|_{s=-s_{0}} \\
& a_{-k}=\left.\frac{1}{(n-k) !} \frac{d^{n-k}}{d s^{n-k}}\left[\left(s+s_{0}\right)^{n} f(s)\right]\right|_{s=-s_{0}}
\end{aligned}
$$

For the poles at $\mathbf{s}=0$, the principal part of the Laurent expansion is most easily found by expressing the numerator and denominator of $\mathrm{Y}(\mathrm{s})$ as an infinite series and using the long division algorithm. Not many terms are needed and in many cases only the first term of the numerator and denominator are needed. The examples below will indicate the ease in finding the principal parts of all pertinent poles. 
With $A(s)$ in hand, the periodic part of the solution $P(s)$ can be formed:

$$
\begin{aligned}
& P(s)=Y(s)-A(s) \\
& \frac{P_{0}(s)}{1-e^{-s T}}=\frac{H(s) X_{0}(s)}{1-e^{-s T}}-A(s) \\
& P_{0}(s)=H(s) X_{0}(s)-\left(1-e^{-s T}\right) A(s)
\end{aligned}
$$

This periodic part of $Y(s)$ has only the poles of $\frac{1}{\left(1-e^{-s T}\right)}$ except for the pole at $s=0, i . e$. the periodic poles along the $j \omega$ axis. As defined above $P_{0}(s)$ will transform to $p_{0}(t)$ in the time domain where

$$
p_{0}(t)=\left\{\begin{aligned}
p(t): & 0 \leq t \leq T \\
0: & t<0 \text { and } t>T
\end{aligned}\right.
$$

In numerical solutions, $p(t)$ valid for the first period is all we need since it repeats in all succeeding periods. Thus the above $e^{-s T}$ term can be dropped and we have:

$$
\begin{aligned}
& P_{0}(s)^{\prime}=H(s) X_{0}(s)-A(s) \\
& P_{0}(t)^{\prime}=\mathscr{A}^{-1}\left[P_{0}(s)^{\prime}\right]=P_{0}(t): 0 \leq t \leq T
\end{aligned}
$$

knowing $p_{0}(t)$ ', the peak-to-peak value of ripple may be found. The average value of $\mathrm{p}_{0}(t)^{\prime}$ is zero. Thus the total solution $y(t)$ is:

$$
y(t)=a(t)+p_{0}(t)^{\prime}
$$

where $p_{0}(t)$ ' repeats in every interval of length $T$. In summary, the technique may be formalized as follows: 
1. Find: $x_{0}(s)=\int_{0}^{T} x_{0}(t) e^{-s t} d t$

$$
\text { where } x_{0}(t)=\begin{aligned}
x(t): & 0 \leq t \leq T \\
0: & t<0 \text { and } t>T \text {. }
\end{aligned}
$$

2. Determine the impulse response

$$
H(s)=\frac{Y(s)}{X(s)} \quad \text { of the system. }
$$

3. Form:

$$
Y(s)=H(s) X(s)=\frac{H(s) X_{O}(s)}{1-e^{-s T}}
$$

4. Collect $A(s)$ : the sum of the principal parts of the Laurent expansions of $Y(s)$ about the poles of $H(s)$, $x_{0}(s)$, and the pole of $\frac{1}{\left(1-e^{-s T}\right)}$ at $s=0$.

5. Solve for

$$
P_{0}(s)^{\prime}=H(s) X_{0}(s)-A(s)
$$

6. Transform $A(s)$ and $P_{0}(s)^{\prime}$ to the time domain as $a(t)$ and $p_{0}(t)^{\prime} \quad(0 \leq t \leq T)$

7. The total solution is $y(t)=a(t)+p_{0}(t)^{\prime}$ where $p_{0}(t)$ ' repeats in every interval of length $T$.

\section{Examples of the Method}

Three examples of this technique will be aiven now conforming to the above summary. 
EXAMPLE I. An Integrator Network

1.
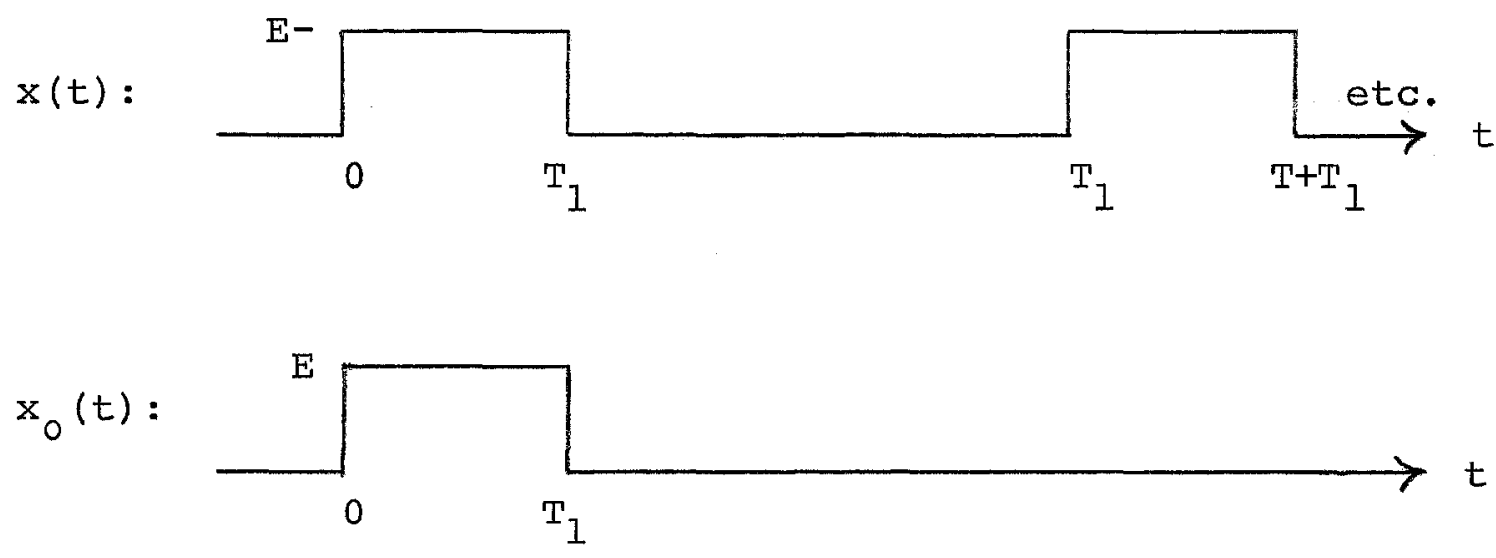

$$
X_{0}(s)=\left(\frac{E}{s}\right)-\left(\frac{E}{s}\right) e^{-s T_{1}}=\frac{E\left(1-e^{-s T_{1}}\right)}{s}
$$

2.

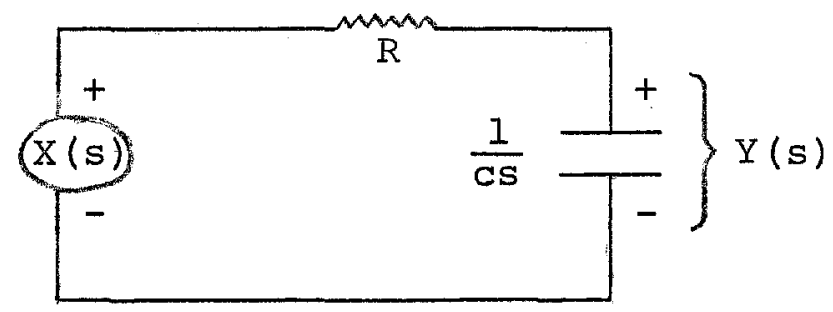

$$
H(s)=\frac{Y(s)}{X(s)}=\frac{\frac{1}{C S}}{\frac{1}{C S}+R}=\frac{\frac{1}{R C}}{\left(s+\frac{1}{R C}\right)}
$$

3.

$$
Y(s)=\frac{H(s) X_{0}(s)}{\left(1-e^{-s T}\right)}=\frac{\left(\frac{E}{R C}\right)\left(1-e^{-s T_{1}}\right)}{s\left(s+\frac{1}{R C}\right)\left(1-e^{-s T}\right)}
$$

where

$$
X(s)=\frac{X_{0}(s)}{\left(1-e^{-s T}\right)}
$$


4. Using the formula for $a_{-1}$ above:

$$
\mathrm{PP}_{1}=\frac{\left(\frac{\mathrm{E}}{\mathrm{RC}}\right)\left(1-\mathrm{e}^{\mathrm{T}_{1} / \mathrm{RC}}\right)}{\mathrm{s}=-\frac{1}{\mathrm{RC}}} \quad \frac{1\left(\frac{1}{\mathrm{RC}}\right)\left(1-e^{\mathrm{T} / \mathrm{RC}}\right)\left(\mathrm{s}+\frac{\mathrm{I}}{\mathrm{RC}}\right)}{}
$$

or:

$$
\mathrm{PP}_{1}=-\frac{E\left(e^{\mathrm{T}_{1} / R C_{-1}}\right)}{\left(e^{\mathrm{T} / \mathrm{RC}}-1\right)\left(s+\frac{1}{\mathrm{RC}}\right)}
$$

Expressing the numerator and denominator of $\mathrm{Y}(\mathrm{s})$ as infinite series:

$$
Y(s)=\frac{\left(\frac{E}{R c}\right)\left[T_{I} s-\frac{\left(T_{1} s\right)^{2}}{2 !}+\frac{\left(T_{I} s\right)^{3}}{3 !}-\ldots\right]}{\left(s^{2}+\frac{s}{R C}\right)\left[T s-\frac{(T s)^{2}}{2 !}+\frac{(T S)^{3}}{3 !}-\cdots\right]}
$$

Using the long division algorithm

$$
\begin{aligned}
& \mathrm{Ts}^{2}+\ldots \frac{\mathrm{E}\left(\frac{\mathrm{T}_{1}}{\mathrm{~T}}\right) \frac{1}{\mathrm{~s}}-\ldots .}{\mathrm{E} \pi \mathrm{s}} \\
& \hline \mathrm{ET}_{1} \mathrm{~s}=\ldots \\
& \therefore \mathrm{PP}_{2} \overline{\mathrm{s}=0} \mathrm{E}\left(\frac{\mathrm{T}}{\mathrm{T}}\right) \frac{1}{\mathrm{~S}}
\end{aligned}
$$

Collecting the principal parts:

$$
\left.A(s)=E\left\{\frac{\left(T_{1}\right)}{T}\right) \frac{1}{s}-\frac{\left(e^{T_{1} / R C}-1\right)}{\left(e^{T / R C_{-1}} /\left(s+\frac{1}{R c}\right)\right.}\right\}
$$


5. $\mathrm{P}_{\mathrm{O}}(\mathrm{s})^{\prime}=\mathrm{H}(\mathrm{s}) \mathrm{X}_{\mathrm{O}}(\mathrm{s})-\mathrm{A}(\mathrm{s})$

$P_{0}(s)^{\prime}=E\left\{\frac{\left(\frac{1}{R c}\right)}{s\left(s+\frac{1}{R c}\right)}-\frac{\left(\frac{1}{R c}\right) e^{-s T_{1}}}{s\left(s+\frac{1}{R C}\right)}-\frac{T_{1}}{T s}+\frac{\left(\frac{e^{T / R c}-1}{T_{1} / R c}\right)}{\left(s+\frac{1}{R c}\right)}\right\}$

6. $a(t)=E\left[\left(\frac{T_{1}}{T}\right)-\left(\frac{e^{T_{1} / R_{c}-1}}{e^{T / R c_{1}}}\right) e^{-t / R c}\right]$

$p_{0}(t)^{\prime}=E\left\{\left(1-e^{-t / R c}\right)-\left(1-e^{-\frac{t-T_{1}}{R C}}\right)_{\mu_{-1}}\left(t-T_{1}\right)\right.$

$$
\left.-\left(\frac{T_{1}}{T}\right)+\left(\frac{e^{T_{1} / R C_{-1}}}{e^{T / R C_{-1}}}\right) e^{-\frac{t}{R C}}\right)
$$

where $\mu_{-1}\left(t-T_{1}\right)= \begin{cases}1: & t-T_{1}>0 \\ 0: & t-T_{1}<0\end{cases}$

Using the numerical values:

$$
\begin{aligned}
& \mathrm{T}_{1}=1 ; \mathrm{T}=3 ; \mathrm{E}=1 \mathrm{~V} ; \mathrm{R}=1 \Omega ; \mathrm{C}=1 \mathrm{fd} . \\
& \mathrm{A}(\mathrm{t})=0.33333-0.09003 \mathrm{e}^{-t} \\
& \mathrm{p}_{0}(\mathrm{t})^{\prime}=\left\{\left(1-\mathrm{e}^{-t}\right)-\left[1-\mathrm{e}^{-(t-1)}\right] \mu_{-1}(t-1)-0.33333\right. \\
& \left.+0.09003 \mathrm{e}^{-t}\right\}
\end{aligned}
$$

7. $y(t)=a(t)+p_{0}(t)$

See Fig. 1 for a picture of these results. 
EXAMPLE II. Full Wave Rectification Filter

1 .
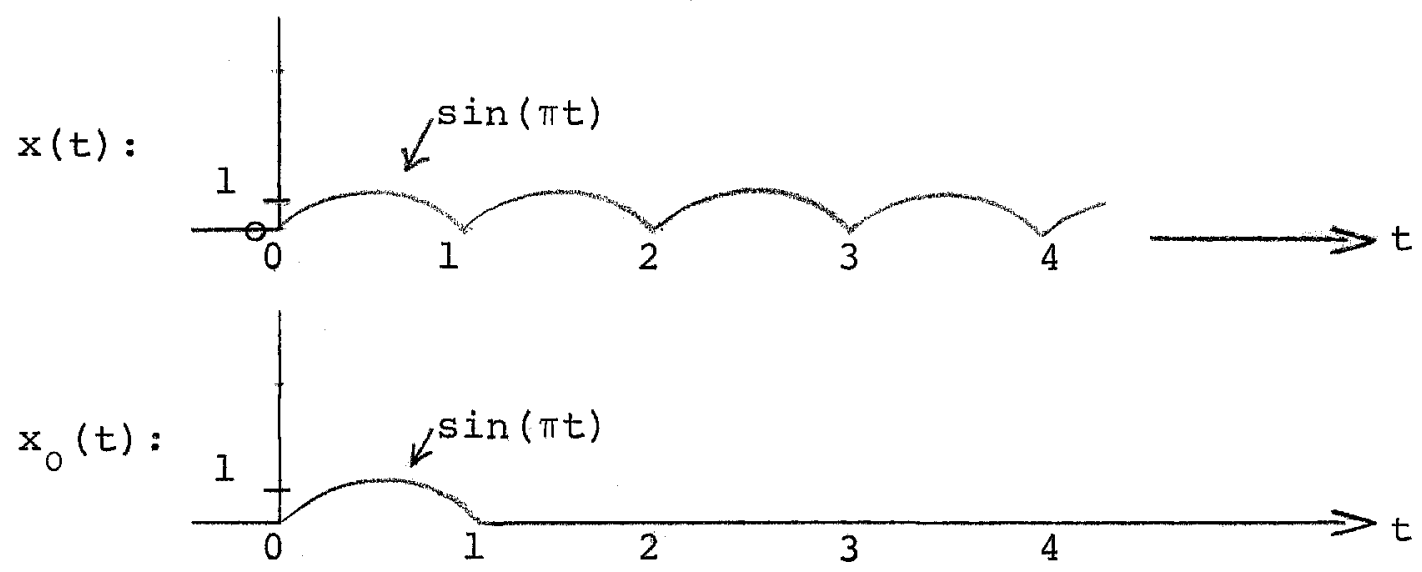

$$
x_{0}(s)=\frac{\pi}{x^{2}+\pi^{2}}+\frac{\pi e^{-s}}{s^{2}+\pi^{2}}=\frac{\pi\left(1+e^{-s}\right)}{\left(s^{2}+\pi^{2}\right)}
$$

2 .

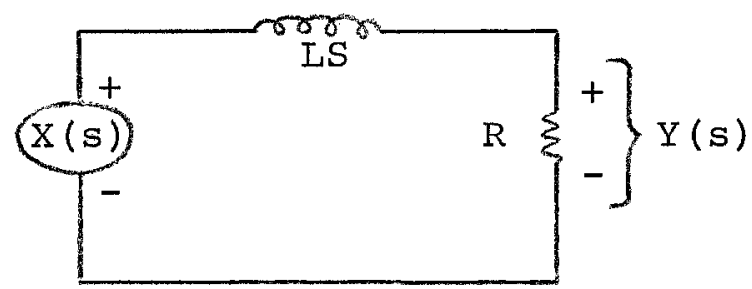

$$
\begin{aligned}
& \mathrm{L}=1 \mathrm{~h} \\
& \mathrm{R}=1 \Omega \\
& \mathrm{T}=1
\end{aligned}
$$

$H(s)=\frac{Y(s)}{X(s)}=\frac{R}{R+L s}=\frac{(R / L)}{(s+R / L)}=\frac{I}{(s+I)}$

3.

$$
\begin{aligned}
& Y(s)=\frac{H(s) X_{0}(s)}{\left(1-e^{-s}\right)}=\frac{\pi\left(1+e^{-s}\right)}{(s+1)\left(s^{2}+\pi^{2}\right)\left(1-e^{-s}\right)} \\
& \text { where } X(s)=\frac{X_{0}(s)}{\left(1-e^{-s}\right)}
\end{aligned}
$$


4. Using the formula for $a_{-1}$ above:

$\mathrm{PP}_{1}=\frac{\pi(e+1)}{\overline{s=-1}}-\frac{\left(\pi^{2}+1\right)(e-1)(s+1)}{\left(\pi^{2}\right.}$

$\mathrm{PP}_{2} \overline{\overline{s=-j \pi}} \frac{\pi\left(e^{j \pi}+1\right)}{(1-j \pi)(-j 2 \pi)\left(1-e^{j \pi}\right)}=0 ; \mathrm{PP}_{3} \overline{\overline{s=+j \pi}}^{\mathrm{s}} 0$

$\mathrm{PP}_{2}$ and $\mathrm{PP}_{3}$ are zero because of the pole zero cancellation at these points. Expressing the numerator and denominator of $Y(s)$ as infinite series:

$Y(s)=\frac{\pi\left[1+1-s+\frac{s^{2}}{2 !}-\cdots\right]}{\left(s^{3}+s^{2}+\pi^{2} s+\pi^{2}\right)\left[s-\frac{s^{2}}{2 !}+\frac{s^{3}}{3 !}-\cdots\right]}$

Using the long division algorithm:

$$
\begin{aligned}
& {\left[\pi^{2} \mathrm{~s}+\cdots\right] \sqrt{\left.\frac{(2}{\pi}\right) \frac{1}{\mathrm{~s}}+\cdots} \sqrt{\frac{[2 \pi}{2 \pi}}} \\
& \therefore \mathrm{PP}_{4 \overline{\mathrm{s}=0}}\left(\frac{2}{\pi}\right) \frac{1}{\mathrm{~s}}
\end{aligned}
$$

Collecting the principal parts:

$$
A(s)=-\frac{\pi(e+1)}{\left(\pi^{2}+1\right)(e-1)(s+1)}+\left(\frac{2}{\pi}\right) \frac{1}{s}
$$


5. $P_{0}(s)^{\prime}=H(s) X_{0}(s)-A(s)$

$$
P_{0}(s)^{\prime}=\frac{\pi\left(1+e^{-s}\right)}{(s+1)\left(s^{2}+\pi^{2}\right)}+\frac{\pi(e+1)}{\left(\pi^{2}+1\right)(e-1)}-\frac{(2 / \pi)}{s}
$$

At this point it is important to notice that the first term has a shifting function, $e^{-s}$, which places that part of the periodic time domain solution out of the period of interest where $p_{0}(t)$ ' is defined. Therefore, this part of the solution must be neglected before proceeding, i.e.

$$
P_{0}(s)^{\prime}=\frac{\pi}{(s+1)\left(s^{2}+\pi^{2}\right)}+\frac{\pi(e+1)}{\left(\pi^{2}+1\right)(e-1)}-\frac{(2 / \pi)}{s}
$$

Now expressing the first term in partial fractions yields:

$P_{0}(s)^{\prime}=\frac{\left(\frac{\pi}{\pi^{2}+1}\right)}{(s+1)}+\frac{1}{\frac{2(-\pi-j)}{(s+j \pi)}}+\frac{\frac{1}{2(-\pi+j)}}{(s-j \pi)}+\frac{\pi(e+1)}{\left(\pi^{2}+1\right)(e-1)(s+1)}-\frac{2 / \pi}{s}$

or

$$
P_{0}(s)^{\prime}=\frac{2 \pi e}{\left(\pi^{2}+1\right)(e-1)(s+1)}-\frac{2 / \pi}{s}+\frac{\pi}{\left(\pi^{2}+1\right)\left(s^{2}+\pi^{2}\right)}-\frac{s \pi}{\left(\pi^{2}+1\right)\left(s^{2}+\pi^{2}\right)}
$$

6.

$$
\begin{aligned}
& a(t)=-\frac{\pi(e+1) e^{-t}}{\left(\pi^{2}+1\right)(e-1)}+\frac{2}{\pi} \\
& p_{0}(t)^{\prime}=\frac{2 \pi e e^{-t}}{\left(\pi^{2}+1\right)(e-1)}-\left(\frac{2}{\pi}\right)+\frac{\sin (\pi t)}{\left(\pi^{2}+1\right)}-\frac{\pi \cos (\pi t)}{\left(\pi^{2}+1\right)}
\end{aligned}
$$


Evaluating the constants yields:

$$
\begin{aligned}
a(t)= & -0.62544 e^{-t}+0.63662 \\
p_{0}(t)^{\prime}=\left\{0.91446 e^{-t}\right. & -0.63662+0.09200 \sin (\pi t) \\
& -0.28903 \cos (\pi t)\}
\end{aligned}
$$

See Figure 2 for a picture of these results.

EXAMPLE III. Approximation of a 6-pulse SCR Power Supply Operating in the constant current Mode and Driving an Inductive Load

1.
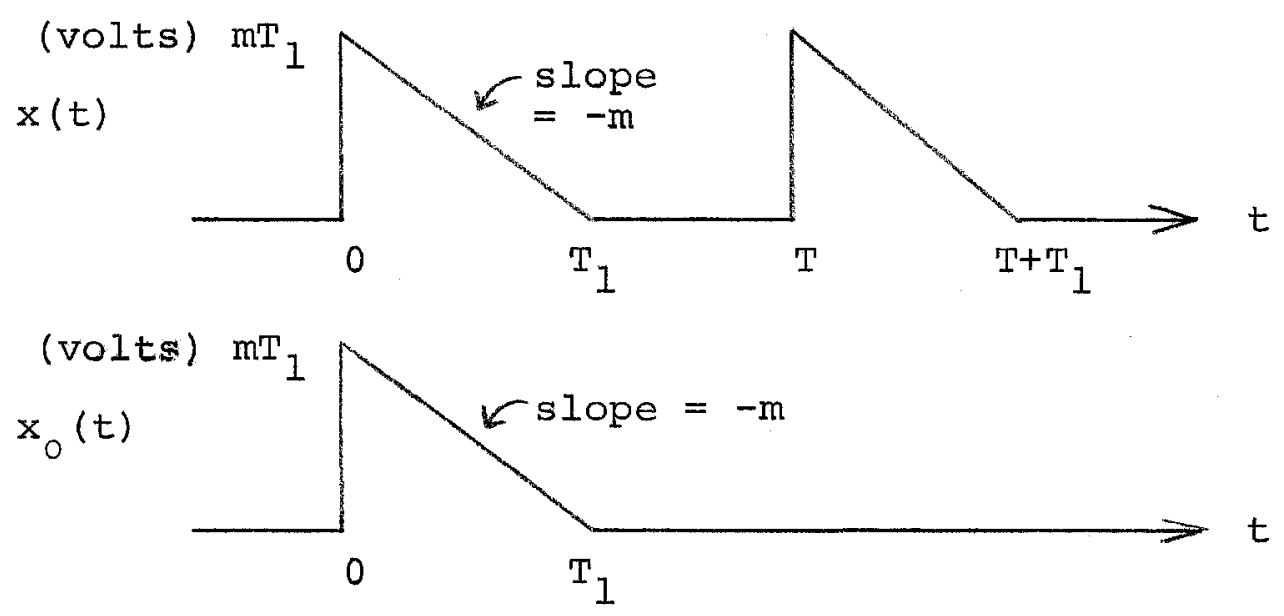

Note: The approximation in this problem is in assuming that the small portion of the sine wave shown above has a constant slope of $-\mathrm{m}$.

$$
\begin{aligned}
& \mathrm{x}_{0}(\mathrm{t})=\mathrm{mT}_{1}-\mathrm{mt}+\mathrm{m}\left(\mathrm{t}-\mathrm{T}_{1}\right) \mu_{-1}\left(t-\mathrm{T}_{1}\right) \\
& \mathrm{x}_{0}(\mathrm{~s})=\frac{\mathrm{mT}_{1}}{\mathrm{~s}}-\frac{\mathrm{m}}{\mathrm{s}^{2}}+\frac{\mathrm{me} \mathrm{sT}_{1}}{\mathrm{~s}^{2}}=\frac{\mathrm{mT}_{1}}{\mathrm{~s}}-\frac{\mathrm{m}}{\mathrm{s}^{2}}\left(1-\mathrm{e}^{-\mathrm{sT}} 1\right)
\end{aligned}
$$


2.

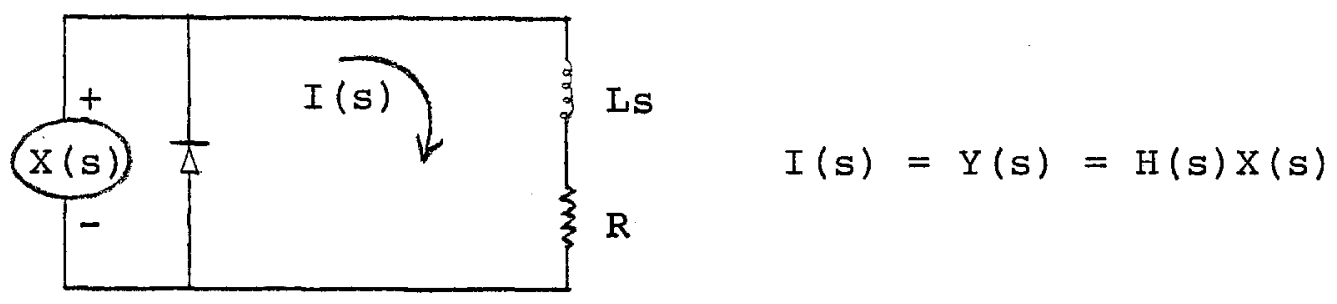

$$
H(s)=\frac{1}{L s+R}=\frac{1}{L\left(s+\frac{R}{L}\right)}=\left(\frac{I}{R}\right) \frac{\left(\frac{R}{L}\right)}{\left(s+\frac{R}{L}\right)}
$$

Let: $\frac{L}{R}=\tau$

$$
\text { i.e. } \quad H(s)=\left(\frac{1}{R}\right) \frac{\left(\frac{1}{\tau}\right)}{\left(s+\frac{1}{\tau}\right)}
$$

3.

$$
Y(s)=\frac{H(s) X_{0}(s)}{\left(1-e^{-s T}\right)}=\left(\frac{m}{R}\right)\left\{\frac{\left(\frac{1}{\tau}\right)\left[\frac{T_{1}}{s}-\frac{\left(1-e^{-s T_{1}}\right)}{s^{2}}\right]}{\left(s+\frac{1}{\tau}\right)\left(1-e^{-s T}\right)}\right\}
$$

or

$$
Y(s)=\left(\frac{m}{R}\right)\left\{\frac{\left(\frac{T_{1}}{\tau}\right)}{s\left(s+\frac{1}{\tau}\right)\left(1-e^{-s T}\right)}-\frac{\left(\frac{1}{\tau}\right)\left(1-e^{-\mathrm{sT}_{1}}\right)}{s^{2}\left(s+\frac{1}{\tau}\right)\left(1-e^{-s T}\right)}\right\}
$$

4. Using the formula for $a_{-1}$ above:

$$
\left.\operatorname{PP}_{1}=\frac{\left(\frac{\mathrm{T}_{1}}{\mathrm{~s}=-1 / \tau}\right)}{\mathrm{R}}\right)\left\{\frac{\mathrm{m}}{-\left(\frac{1}{\tau}\right)\left(1-\mathrm{e}^{\mathrm{T} / \tau}\right)\left(\mathrm{s}+\frac{1}{\tau}\right)}-\frac{\left(\frac{1}{\tau}\right)\left(1-e^{\mathrm{T}_{1} / \tau}\right)}{\left(\frac{1}{\tau}\right)^{2}\left(1-e^{\mathrm{T} / \tau}\right)\left(s+\frac{1}{\tau}\right)}\right\}
$$


or $\quad \mathrm{PP}_{1}=\left(\frac{m}{\mathrm{R}}\right) \frac{\left[\mathrm{T}_{1}-\tau\left(e^{\mathrm{T}_{1} / \tau}-1\right)\right]}{\left(e^{\mathrm{T} / \tau}-1\right)\left(s+\frac{1}{\tau}\right)}$

Expressing the numerator and denominator of $Y(s)$ as infinite series:

$Y(s)=\left(\frac{m}{R}\right)\left\{\frac{\left(T_{I} / \tau\right)}{\left(s^{2}+\frac{s}{\tau}\right)\left[T s-\frac{(T s)^{2}}{2 !}+\cdots\right]}-\frac{\left(\frac{I}{\tau}\right)\left[T_{I} s-\frac{\left(T_{I} s\right)^{2}}{2 !}+\cdots\right]}{\left(s^{3}+\frac{s^{2}}{\tau}\right)\left[T s-\frac{(T s)^{2}}{2 !}+\cdots\right]}\right\}$

or

$Y(s)=\left(\frac{m}{R}\right)\left\{\frac{\left(T_{I} / \tau\right)}{\left[\left(\frac{T}{\tau}\right) s^{2}+\left(T-\frac{T}{2 \tau}\right) s^{3} \ldots \ldots\right]}-\frac{\frac{1}{\tau}\left[T_{1} s-\frac{\left(T_{I} s\right)^{2}}{2 !}+\cdots\right]}{\left[\left(\frac{T}{\tau}\right) s^{3}+\left(T-\frac{T^{2}}{2 \tau}\right) s^{4} \ldots\right]}\right\}$

Using the long division algorithm twice:

$$
\frac{\left.\left[\left(\frac{T}{\tau}\right) s^{2}+\left(T-\frac{T^{2}}{2 \tau}\right) s^{3} \cdots\right]\right]}{\frac{\left(\frac{T}{T}\right) \frac{1}{s^{2}}-\left(\frac{\tau}{T}\right)^{2} \cdot\left[T-\frac{T^{2}}{2 \tau}\right] \frac{1}{S}+\cdots}{\frac{1}{1+\left(\frac{\tau}{T}\right)\left(T-\frac{T}{2 \tau}\right) s-\cdots}}} \frac{-\left(\frac{\tau}{T}\right)\left(T-\frac{T^{2}}{2 \tau}\right) s+\cdots}{\left(\frac{\tau}{T}\right)\left(T-\frac{T^{2}}{2 \tau}\right) s-\cdots}
$$




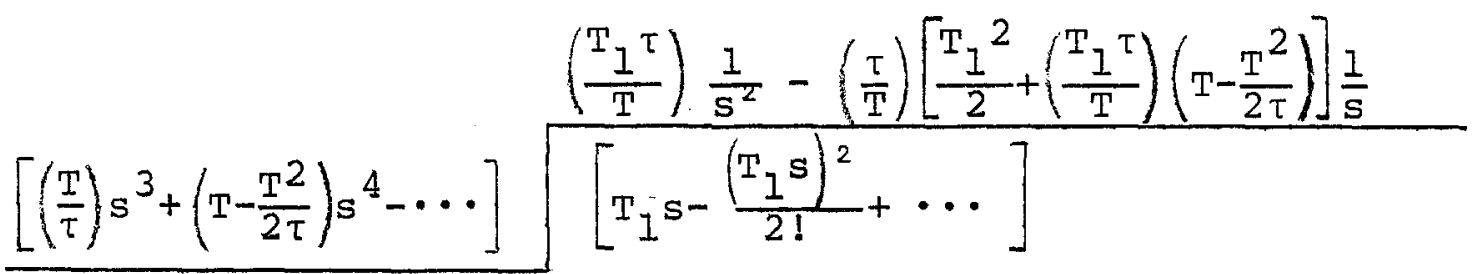

$$
\begin{aligned}
& \frac{T_{1} s+\left(\frac{T_{1}^{T}}{T}\right)\left(T-\frac{T^{2}}{2 \tau}\right) s^{2}-\cdots}{-\left[\frac{T_{1}^{2}}{2}+\left(\frac{T_{1}{ }^{T}}{T}\right)\left(T-\frac{T^{2}}{2 \tau}\right)\right] s^{2}+\cdots} \\
& -\left[\frac{\mathrm{T}_{1}{ }^{2}}{2}+\left(\frac{\mathrm{T}_{1}{ }^{\tau}}{\mathrm{T}}\right)\left(\mathrm{T}-\frac{\mathrm{T}^{2}}{2 \tau}\right)\right] \mathrm{s}^{2} \ldots
\end{aligned}
$$

$$
\begin{aligned}
\therefore P_{2}=\left(\frac{m}{\bar{R}}\right)\left\{\left(\frac{T}{\tau}\right)\left[\left(\frac{\tau}{T}\right) \frac{1}{s^{2}}-\left(\frac{\tau}{T}\right)^{2}\left(T-\frac{T^{2}}{2 \tau}\right) \frac{1}{s}\right]\right. \\
\left.\left(\frac{1}{\tau}\right)\left[\left(\frac{T_{I} \tau}{T}\right) \frac{1}{s^{2}}-\left(\frac{\tau}{T}\right)\left(\frac{T_{1}^{2}}{2}+\left(\frac{T_{1} \tau}{T}\right)\left(T-\frac{T^{2}}{2 \tau}\right)\right) \frac{I}{s}\right]\right\}
\end{aligned}
$$

or $\mathrm{PP}_{2}=\left(\frac{\mathrm{m}}{\mathrm{R}}\right)\left(\frac{\mathrm{T}_{1}{ }^{2}}{2 \mathrm{~T}}\right) \frac{1}{\mathrm{~s}}$

Collecting the principal parts:

$$
A(s)=\left(\frac{m}{R}\right)\left\{\left(\frac{T_{1}^{2}}{2 T}\right) \frac{1}{s}-\frac{\left[\tau\left(e^{T_{1} / \tau}-1\right)-T_{1}\right]}{\left(e^{T / \tau}-1\right)\left(s+\frac{1}{\tau}\right)}\right\}
$$

5. $P_{0}(s)^{\prime}=H(s) X_{0}(s)-A(s)$

$$
P_{0}(s)^{\prime}=\left(\frac{m}{R}\right)\left\{\frac{T_{1} / \tau}{s\left(s+\frac{1}{\tau}\right)}-\frac{\left(\frac{1}{\tau}\right)\left(1-e^{-s T_{1}}\right)}{s^{2}\left(s+\frac{1}{\tau}\right)}+\frac{\left[\tau\left(e^{T_{1} / \tau}-1\right)-T_{1}\right]}{\left(e^{T / \tau}-1\right)\left(s+\frac{1}{\tau}\right)}-\left(\frac{T_{1}{ }^{2}}{2 T}\right) \frac{1}{s}\right\}
$$


6.

$$
\begin{aligned}
& a(t)=\left(\frac{m}{R}\right)\left\{\frac{T_{1}{ }^{2}}{2 T}-\frac{\left[\tau\left(e^{T_{1} / \tau}-I\right)-T_{1}\right] e^{-t / \tau}}{\left(e^{T / \tau}-I\right)}\right\} \\
& p_{0}(t)^{\prime}=\left(\frac{m}{R}\right)\left\{T_{1}\left(1-e^{-t / \tau}\right)-\left[t-\tau\left(1-e^{-t / \tau}\right)\right]\right. \\
& +\left[\left(t-T_{1}\right)-\tau\left(1-e^{\frac{-\left(t-T_{1}\right)}{\tau}}\right)\right]_{\mu_{-1}\left(t-T_{1}\right)} \\
& \left.+\frac{\left[\tau\left(e^{T_{1} / \tau}-I\right)-T_{1}\right] e^{-t / \tau}}{\left(e^{T / \tau}-1\right)}-\frac{T_{1}^{2}}{2 T}\right)
\end{aligned}
$$

7. $y(t)=a(t)+p_{0}(t)$

In order to find the maximum value of $\mathrm{p}_{0}(t)$ ' for $0<t<\mathrm{T}_{1}$ ' the derivative of $p_{0}(t)$ ' will be taken:

$$
\frac{d\left[p_{0}(t)^{\prime}\right]}{d t}=\left(\frac{m}{R}\right)\left\{\left[\left(\frac{T_{1}}{\tau}\right)+1-\left(\frac{e^{T_{1} / \tau}-1-\frac{T_{1}}{\tau}}{e^{T / \tau}-I}\right)\right] e^{-t / \tau}-1\right\}
$$

setting the derivative equal to zero and solving for $t_{\max }$ yields:

$$
t_{\max }=\tau \ln \left\{\left(\frac{\mathrm{T}_{1}}{\tau}\right)+1-\left(\frac{e^{\mathrm{T}_{1} / \tau}-1-\frac{\mathrm{T}_{1}}{\tau}}{e^{\mathrm{T} /} \tau_{-1}}\right)\right\}
$$


Since this particular example was the "raison d'etre" of this work, two numerical substitutions will be made: Case A using simple numbers with $T_{1}$ as a parameter and Case $B$ using the actual values encountered in a 6-pulse SCR-power supply driving a constant current into a magnet load. In Case B $\mathrm{T}_{1}=\mathrm{T}$ for maximum ripple current on the output.

See Fig. 3 for a picture of Case A where: $\mathrm{L}=1 \mathrm{hy}$, $\mathrm{R}=1 \Omega, \mathrm{m}=1, \tau=1, \mathrm{~T}=2$, i.e.

$$
\begin{aligned}
p_{0}(t)^{\prime}= & \left\{T_{1}\left(1-e^{-t}\right)-\left[t-\left(1-e^{-t}\right)\right]\right. \\
& +\left[\left(t-T_{1}\right)-\left(1-e^{-\left(t-T_{1}\right)}\right)\right] \mu_{-1}\left(t-T_{1}\right) \\
& \left.+\frac{\left[\left(e^{T_{1}}\right)-T_{1}\right]}{\left(e^{2}-1\right)}-\frac{T_{1}^{2}}{4}\right\}
\end{aligned}
$$

Case B involved an Acme 6-pulse SCR-power supply driving a quadrupole magnet in the current mode. The worst case driving voltage waveform looked as follows:

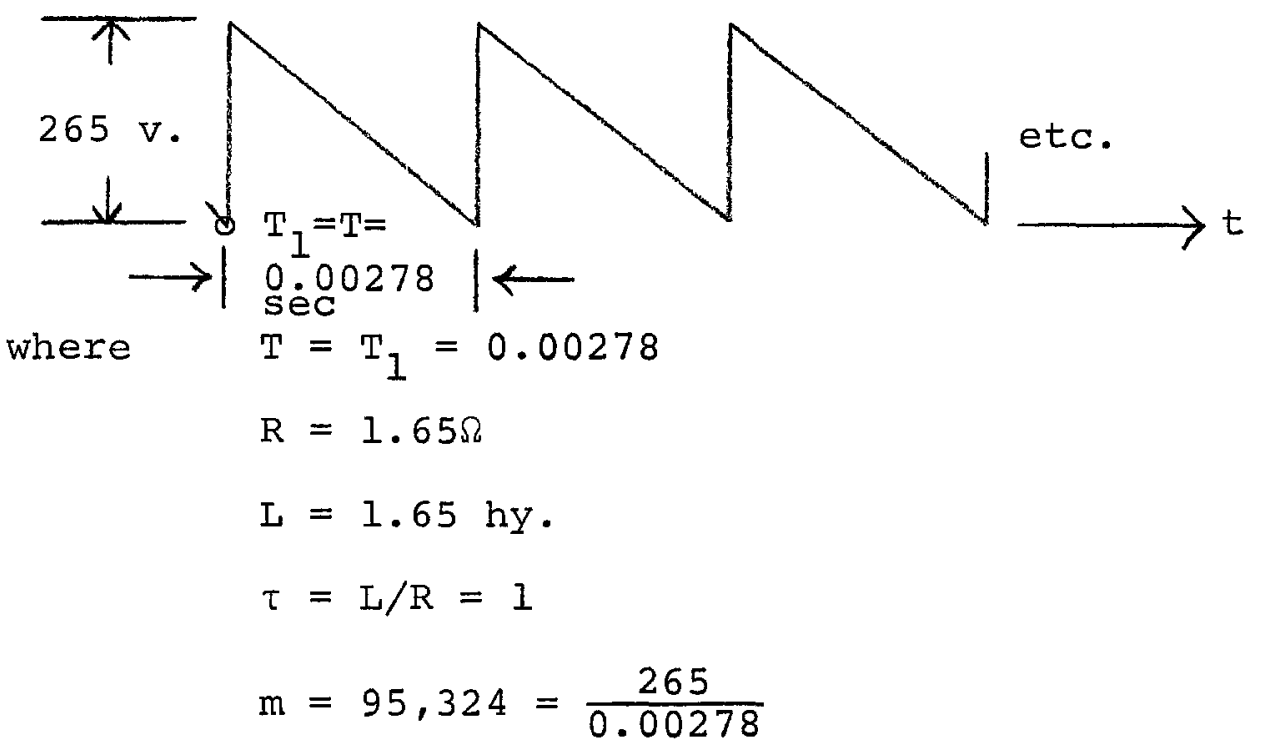


The results of substituting these values in the general equations above are:

$$
\begin{array}{ll}
a(\infty) & =80.3030 \mathrm{amp} \\
\mathrm{p}_{0}(0)^{\prime} & =\mathrm{p}_{0}\left(\mathrm{~T}_{I}\right)^{\prime}=-0.0372 \mathrm{amp} \\
t_{\max } & =0.0013897 \\
\mathrm{p}_{0}\left(t_{\max }\right)^{\prime} & =+0.0186 \mathrm{amp}
\end{array}
$$

peak-to-peak ripple $=p_{0}\left(t_{\max }\right)^{\prime}-p_{0}(0)^{\prime}=0.0558 \mathrm{amp}$

It will be noted that the maximum value occurs almost at the midpoint of the period $T$ so that the actual ripple output current must be approximately symmetrical about the value $\mathrm{T} / 2$.

\section{Acknowledgement}

This work is patterned after a section of LePage's book, "Complex Variables and Laplace Transforms" 1961. However, he excludes from consideration the common case where poles of $H(s) X_{0}(s)$ coincide with roots of $\left(1-e^{-s T}\right)$, which are covered here in the examples. 
EXAMPLE I THE COMPLETE SOLUTION

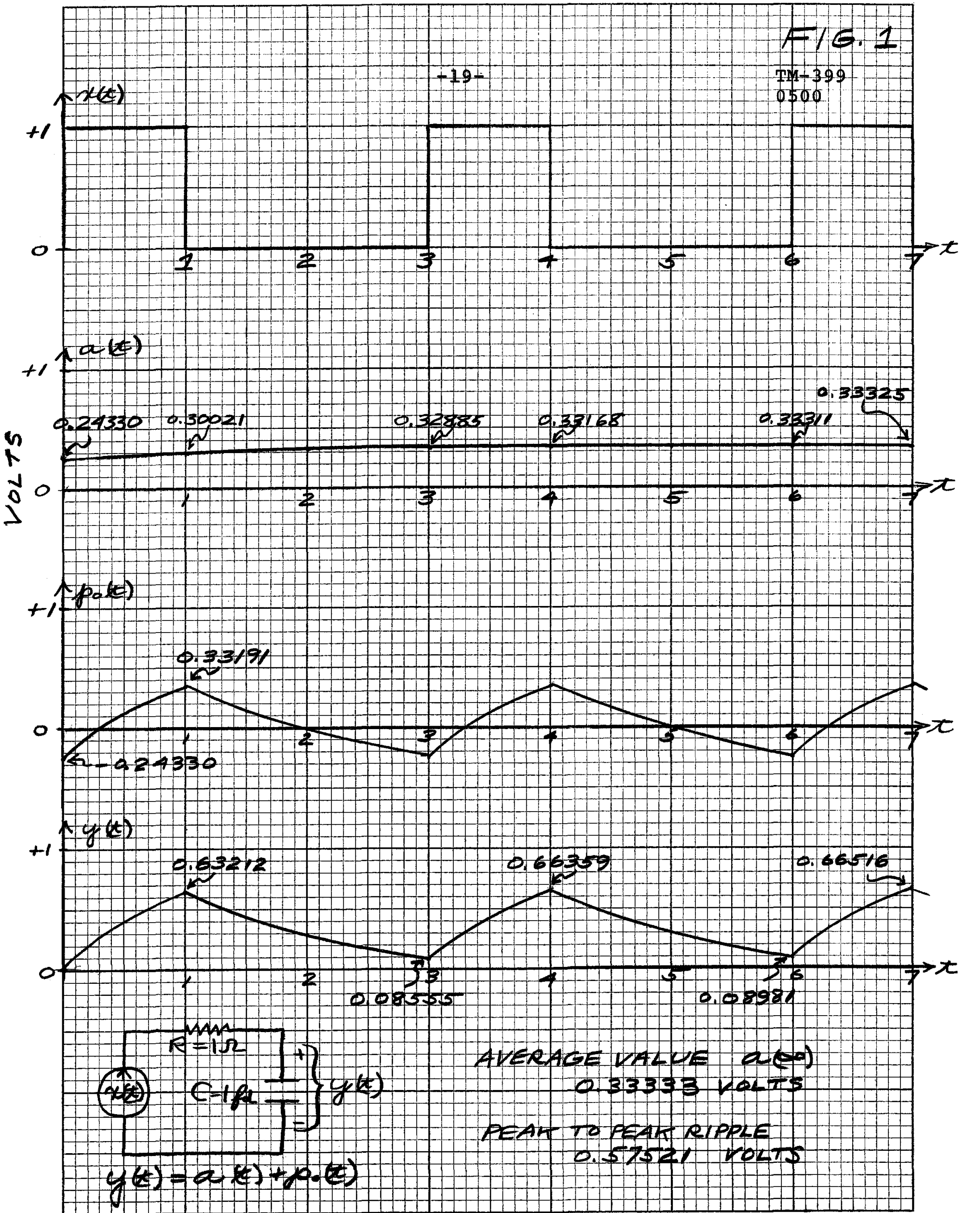




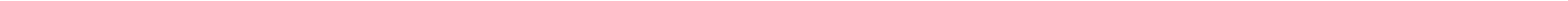

